\title{
A Tale of Diagnostic Delay with Detrimental Consequences: Illustrating the Challenging Nature of Diagnosing Giant Cell Arteritis
}

\author{
Marieke van Nieuwland $^{1}$, Dennis Boumans ${ }^{1}$, Gerben Jan Jacob Plas ${ }^{2}$, Onno Dirk Vijlbrief ${ }^{3}$, Celina Alves ${ }^{1}$ \\ ${ }^{1}$ Department of Rheumatology and Clinical Immunology, Ziekenhuisgroep Twente (Hospital Group Twente), Almelo, The Netherlands \\ ${ }^{2}$ Department of Neurology, Ziekenhuisgroep Twente (Hospital Group Twente), Almelo, The Netherlands \\ ${ }^{3}$ Department of Radiology, Ziekenhuisgroep Twente (Hospital Group Twente), Almelo, The Netherlands
}

\section{Doi: 10.12890/2021_002562 - European Journal of Case Reports in Internal Medicine - @ EFIM 2021}

Received: $14 / 04 / 2021$

Accepted: 03/06/2021

Published: $27 / 07 / 2021$

\begin{abstract}
How to cite this article: van Nieuwland M, Boumans D, Plas GJJ, Vijlbrief OD, Alves C. A tale of diagnostic delay with detrimental consequences: illustrating the challenging nature of diagnosing giant cell arteritis.EJCRIM 2021;8: doi:10.12890/2021_002562.
\end{abstract}

Conflicts of Interests: The authors declare there are no competing interests.

This article is licensed under a Commons Attribution Non-Commercial 4.0 License

\section{ABSTRACT}

Giant cell arteritis is a medical emergency as severe, irreversible complications may occur if it is not treated in a timely manner. However, in daily practice early diagnosis can be challenging. We report the case of a 70-year-old woman who presented with multiple ischaemic cerebral vascular accidents related to newly diagnosed giant cell arteritis. Review of her charts revealed a substantial delay from the onset of symptoms to diagnosis. This case demonstrates the need for additional efforts to reduce delay in referring patients with giant cell arteritis and the need to implement fast-track clinics to prevent serious complications.

\section{LEARNING POINTS}

- Giant cell arteritis is a medical emergency and unnecessary diagnostic delay can result in severe complications.

- Despite implementation of fast-track clinics, diagnostic delay still occurs due to the generic nature of signs and symptoms and inadequate case finding.

- As diagnostic delay can lead to preventable complications, increased knowledge and awareness of the characteristics and urgency of giant cell arteritis is needed among referring physicians.

\section{KEYWORDS}

Giant cell arteritis, diagnostic delay, severe complications

\section{CASE DESCRIPTION}

A 70-year-old woman presented to the internal medicine outpatient department with significant malaise, a $7 \mathrm{~kg}$ weight loss in approximately 8 months, alternating parietal headache for 5 months, and transient neck pain during a 4-week period which subsided with physical therapy. After onset of the headache, the patient had consulted her general practitioner. She had also visited an ophthalmologist with visual complaints without vision loss in the left eye. Fundoscopy showed cotton wool spots, but details of this examination were not available to us. Laboratory assessment 2 weeks before visiting the internal medicine outpatient department showed an elevated C-reactive protein (CRP; 126 mg/l) and erythrocyte sedimentation rate (ESR; $130 \mathrm{~mm} / \mathrm{h}$ ). The patient went home pending additional diagnostic testing (laboratory assessment and computed tomography (CT)), with multiple differential diagnoses including malignancy, infection and vasculitis. Eight days later, she presented to the emergency department with increased malaise, transient dizziness and coordination disturbance. CRP was still elevated $(148 \mathrm{mg} / \mathrm{l})$. She was discharged after acute neurological pathology was ruled with CT of the brain. Another 8 days later, she again visited the 
internal medicine outpatient department in a deteriorated condition with neurological symptoms warranting referral to a neurologist. She was admitted to the neurocritical care unit because of diplopia with a clinical picture of wall-eyed bilateral internuclear ophthalmoplegia and dysarthria. The rheumatology giant cell arteritis fast-track clinic was immediately consulted for vasculitis work-up including colour duplex ultrasound ${ }^{[1]}$.

After fast-track clinic consultation, giant cell arteritis was promptly diagnosed using ultrasound, which showed a positive diffuse halo sign (including positive compression signs) and increased intima-media thickness (above the cut-off values) of the axillary, facial and superficial temporal arteries including their branches (Fig. 1) ${ }^{[2]}$.
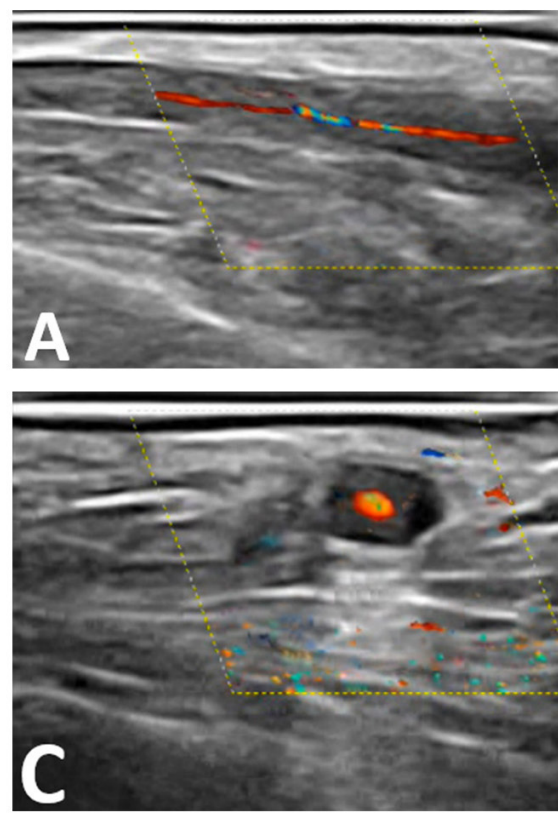
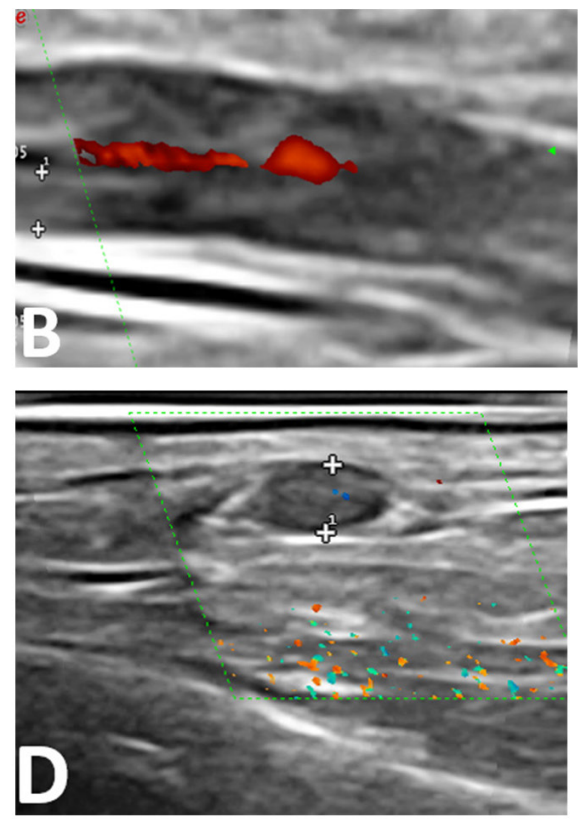

Figure 1. Longitudinal $(A+B)$ and transversal $(C+D)$ ultrasound images $(22 \mathrm{MHz})$ of the superficial temporal artery. A positive halo sign $(A-C)$ and compression sign (D) were captured. An increased intima-media thickness of $0.61 \mathrm{~mm}$ longitudinal (B), and artery compression diameter of $1.5 \mathrm{~mm}$ transversal (D) was measured between the measuring points (++)

Several temporal artery branches also showed stenosis and occlusions. The abdominal and chest CT, performed shortly before admission, showed a slightly thickened aorta and iliac artery vessel wall (Fig. 2).

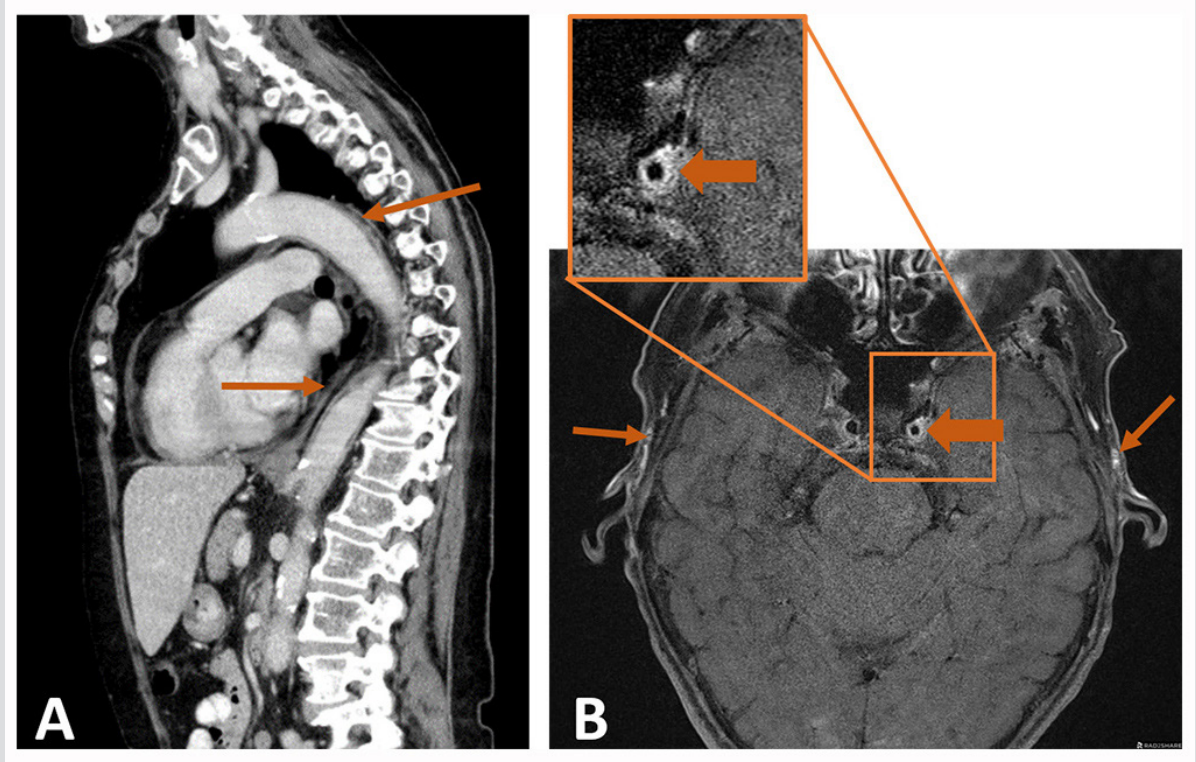

Figure 2. CT and MRI imaging results supporting the diagnosis. (A) Sagittal plane of the abdominal and chest CT showing a slightly increased aorta vessel wall without an aneurysm. (B) Brain MRI (Ax T1 fs SE 3mm) showing irregular arterial wall enhancement of the left internal carotid artery (large arrows) and both common superficial temporal arteries (small arrows) 
Brain magnetic resonance imaging (MRI) with contrast, performed the same day as ultrasound, showed multiple ischaemic lesions (bilateral cerebellar, occipital and one-sided mesencephalic) and a left-sided vertebral artery occlusion. Also, arterial wall enhancement of both common temporal arteries and the left carotid artery was observed on MRI (Fig. 2).

The ultrasound, CT and MRI findings confirmed the final diagnosis of cranial and large vessel giant cell arteritis with secondary multiple bilateral cerebellar, occipital and mesencephalic ischaemic cerebral vascular accidents. Treatment with methylprednisolone (1000 mg/ day for 3 days) followed by prednisolone ( $60 \mathrm{mg} /$ day in a tapering schedule) was immediately started. The patient was discharged 7 days after admission. One month after admission, diplopia and other symptoms had improved substantially and inflammatory markers were normalized (CRP $7 \mathrm{mg} / \mathrm{l}$ and ESR $26 \mathrm{~mm} / \mathrm{h}$ ).

\section{DISCUSSION}

Giant cell arteritis is a medical emergency as it can cause severe complications such as permanent vision loss if not treated in a timely manner. These complications can be prevented by prompt glucocorticoid treatment. Therefore, swift referral, diagnosis and treatment is essential. This case involved a long diagnostic delay in a patient with giant cell arteritis leading to ischaemic cerebral vascular accidents, highlighting the need for and the difficulty of early diagnosis ${ }^{[3]}$.

The delay in diagnosis in our case can be attributed to different factors. First, delay can occur because a patient does not immediately seek medical attention. Based on the information available, our patient was not keen on visiting a general practitioner with general complaints and only attended after the onset of cranial complaints. Second, delay can occur during the referral of a patient when physicians do not consider giant cell arteritis. This is often due to the generic nature of symptoms. In this particular case, there was an approximately 5-month delay from first consultation to fast-track clinic referral, with multiple specialized physicians involved. Eventually, the fast-track clinic was consulted and a swift diagnosis was made using ultrasound, avoiding further diagnostic delay.

Delay can occur because of unique or atypical manifestations, making recognition and diagnosis of giant cell arteritis difficult. However, in this case, in retrospect noticeable indicators were present at an early stage. One meta-analysis described an average delay of 9 weeks from symptom onset to giant cell arteritis diagnosis, with a 95\% confidence interval of 6.5 to 11.4 weeks. However, patients with a cranial manifestation received a diagnosis after an average of 7.7 weeks (95\% confidence interval 2.7 to 12.8 weeks) ${ }^{[4]}$. Delay was substantially lengthened for our patient.

The introduction of fast-track clinics contributes to a decrease in diagnostic delay, significantly reduces vision loss and is cost-effective ${ }^{[5,6]}$. Red flags to contact our fast-track clinic by phone to assess the urgency of diagnostic work-up include acute vision loss and other symptoms (jaw claudication, scalp tenderness, temporal headache and temporal artery abnormalities) occurring in patients over the age of 50 and in particular in the presence of an elevated CRP and/or ESR. In our hospital, the fast-track clinic is available on a daily basis and vascular ultrasound is immediately available, which is recommended as the first diagnostic test [1]. Nevertheless, this case illustrates that despite having a fast-track clinic, not all forms of delay are resolved and that focussing only on the introduction of these clinics will not suffice. It emphasizes that we should make an effort to improve case finding in order to reduce delay in fast-track clinic referral, thereby highlighting the need for increased knowledge and awareness among general practitioners and other referring physicians that giant cell arteritis is a medical emergency.

\section{REFERENCES}

1. Dejaco C, Ramiro S, Duftner C, Besson FL, Bley TA, Blockmans D, et al. EULAR recommendations for the use of imaging in large vessel vasculitis in clinical practice. Ann Rheum Dis 2018;77(5):636-643.

2. Schäfer VS, Juche A, Ramiro S, Krause A, Schmidt WA. Ultrasound cut-off values for intima-media thickness of temporal, facial and axillary arteries in giant cell arteritis Rheumatology 2017;56(9):1479-1483.

3. Baig IF, Pascoe AR, Kini A, Lee AG. Giant cell arteritis: early diagnosis is key. Eye Brain 2019;11:1-12.

4. Prior JA, Ranjbar H, Belcher J, Mackie SL, Helliwell T, Liddle J, et al. Diagnostic delay for giant cell arteritis - a systematic review and meta-analysis. BMC Med 2017;15(1):120.

5. Diamantopoulos AP, Haugeberg G, Lindland A, Myklebust G. The fast-track ultrasound clinic for early diagnosis of giant cell arteritis significantly reduces permanent visua impairment: towards a more effective strategy to improve clinical outcome in giant cell arteritis? Rheumatology 2016;55(1):66-70.

6. Luqmani R, Lee E, Singh S, Gillett M, Schmidt WA, Bradburn M, et al. The role of ultrasound compared to biopsy of temporal arteries in the diagnosis and treatment of giant cell arteritis (TABUL): a diagnostic accuracy and cost-effectiveness study. Health Technol Assess 2016;20(90):1-238. 